\title{
Perioperative surgical home: a new scope for future anesthesiology
}

\author{
Min A Kwon \\ Department of Anesthesiology and Pain Medicine, Dankook University Hospital, Cheonan, Korea
}

The health care system is changing from 'pay for volume' to 'pay for value.' These changes are turning health care delivery into a more cost-effective and coordinated care setup that drives hospitals to lower costs and greater quality gains. The present perioperative care service in Korea has proven to be costly, fragmented, and neither evidence-based nor patient-centered. Recently, a new concept of a perioperative care model termed perioperative surgical home (PSH) has been proposed. The PSH is a patient-centered, team-based, and coordinated perioperative care setup, composed of the head anesthesiologist-perioperativist in tandem with dedicated nurse practitioners and other PSH team doctors. All pre-, intra-, and postoperative patient care functions are performed by a single PSH team, not several different departments. The PSH care extends from the decision to operate till 30 days post-discharge. Several evidence-driven perioperative strategies for reducing postoperative complications and shortening hospital stay can be adapted to each specific hospital situation, rather than strictly applying any given strategies. With the PSH, patients are more satisfied and experience better outcomes. It is also a good hospital business model. The expanded role of anesthesiologists in the PSH has the potential to invigorate the specialty.

Keywords: Delivery of health care; Health care costs; Length of stay; Medical errors; Perioperative care; Value-based purchasing.

\section{Background}

Perioperative care is a challenge to modern medicine, which does not, at present, improve quality despite high costs [1]. The current perioperative care system in Korea is offered in a fragmentary manner by each hospital department and is not patient-centered. Absence of proper communication often results in unnecessary or redundant preoperative testing, delayed

Corresponding author: Min A Kwon, M.D., Ph.D.

Department of Anesthesiology and Pain Medicine, Dankook University Hospital, 201, Manghyang-ro, Dongnam-gu, Cheonan 31116, Korea

Tel: 82-41-550-6814, Fax: 82-41-551-9330

Email:mmauss73@gmail.com

ORCID: https://orcid.org/0000-0002-7253-3768

Received: July 26, 2017.

Revised: November 23, 2017.

Accepted: December 10, 2017.

Korean J Anesthesiol 2018 June 71(3): 175-181

https://doi.org/10.4097/kja.d.18.27182 perioperative care, and frequent errors or accidents [2].

The role of anesthesiologists has often been limited to the period from pre-anesthetic examination just before surgery to the postoperative care unit, or a short period in the ward. Most of the preoperative preparation and postoperative and post-discharge care of the patient have usually been provided by the surgical team.

Anesthesiology has an outstanding record in improving the safety of and reducing avoidable harm to the patient $[3,4]$. The mortality rate directly attributed to anesthesia is very low ( $<1 / 50,000$ mortalities), but the incidence of postoperative complications, at $20-30 \%$ of cases, is still high. In addition, while the incidence of postoperative complications is similar across countries and hospitals, patient outcomes and mortality differ significantly. This suggests that the method of preventing and treating postoperative complications is very different for each institution $[5,6]$. Patients undergoing emergency surgery, or those with severe diseases particularly, show more striking variations in outcomes and mortality rates $[2,6]$.

Accumulating evidence favors a variety of discrete or bun-

(c) This is an open-access article distributed under the terms of the Creative Commons Attribution Non-Commercial License (http://creativecommons.org/ licenses/by-nc/4.0/), which permits unrestricted non-commercial use, distribution, and reproduction in any medium, provided the original work is properly cited. 
dled perioperative interventions like normothermia [7], use of regional anesthesia, avoidance of opioids [8], and enhanced recovery after surgery (ERAS) $[9,10]$ to reduce postoperative complications. Recently, a new concept of perioperative care, termed perioperative surgical home (PSH), has been proposed. The model has been strongly supported by American Society of Anesthesiologists (ASA) [10,11].

In this paper, we present the concept of PSH and historical improvements in perioperative care and discuss how to develop this new paradigm of anesthesiologist-perioperativist in practice, considering the perspective of surgeons, anesthesiologists, and hospital administrators.

\section{The PSH Model}

The average cost of surgery continues to increase, but the outcome and the rate of readmission have not decreased [1]. This is because surgical care is not standardized and coordinated, leading to unnecessary or redundant preoperative testing and procedures [12-14].

For decades, various perioperative care concepts have been developed and reported. In many countries, the health care system is moving from 'pay for volume' to 'pay for value' [15]. These changes are turning health care delivery into a more cost-effective, coordinated care setup that drives hospitals to lower costs and greater quality gains. In this new health care system, the anesthesiologist and other health care providers will be paid according to how well they provide the service consistently, with standardized metrics, not the amount of services provided [15]. In particular, many perioperative complications are caused by the lack of communication and wide variability in the treatment protocols offered by several departments [11], which argue for a new perioperative model.

Postoperative recovery has evolved considerably with the development of several evidence-based treatment options and standardized treatment protocols [8]. The most notable perioperative concept is ERAS to achieve fast-track postoperative patient recovery [16]. The ERAS protocol was first described in the 1990s [10]. It was initially developed for a few colorectal surgery patients but was later extended to other surgical patients. It has recently been adopted in Europe, Canada, and some American institutions. The ERAS protocol consists of about 20 specific treatment options that reduce patient anxiety by detailed preoperative explanation, reduce preoperative fasting with preoperative carbohydrate loading, reduce opioid use by preference of epidural analgesia, and encourage early feeding and ambulation [8]. The ERAS protocol reduces postoperative complications, enhances patient recovery, and reduces the length of hospital stay and medical costs $[8,9,16,17]$.

Recently, the ASA proposed a new paradigm of standardized, evidence-based perioperative strategies called PSH. The PSH is an innovative surgical care model supported with continuous care and shared decision-making [10]. The PSH is characterized as 'a patient-centered and physician-led, multidisciplinary, teambased system of coordinated care that guides the patient through the entire surgical experience' (Fig. 1) [18]. An anesthesiologist-perioperativist is paired with nurse practitioners to provide consistently more focused and integrated postoperative patient care from the recovery room through the ward to post-discharge care [11]. Moreover, the PSH team works closely with acute pain services to assess the patient's pain and manage it effectively. They also plan pain management in advance so opioids, if required, can be timely converted from intravenous to oral formulations [10,11]. This continuous care enhances satisfaction by greater familiarity and reduced anxiety of the patients and their families.

Conceptually, the PSH model aims to reduce variability throughout the surgical process [10]. Variability increases the likelihood of errors and complications. In a poorly organized center, patients can receive unnecessary tests preoperatively and experience delay in the proper administration of medicines or other postoperative therapeutic measures. Inadequate communication and handover between medical staffs is a frequent cause of postoperative complications [11]. A simple way to reduce variability is to view perioperative care as a continuum, not as separate preoperative, intraoperative, postoperative, and post-discharge episodes. This single perioperative episode lasts from preoperative preparation to 30 days post-discharge [18]. Moreover, several evidence-driven perioperative strategies for reducing postoperative complications and shortening hospital stay can be applied to each specific hospital situation [19]. The overall purpose of PSH is to provide better medical services and improve outcomes while reducing costs. Some innovative hospitals have been applying PSH in their surgical care and have

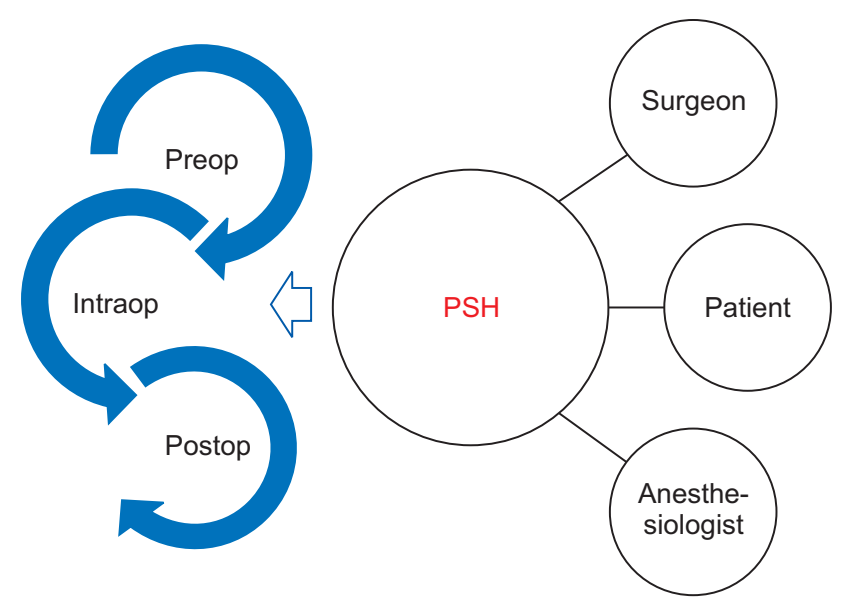

Fig. 1. Perioperative surgical home (PSH). 
managed to effectively reduce hospital stay and medical costs $[11,20,21]$.

\section{Practices of the PSH Model}

During the implementation phase, various departmental experts such as anesthesiologists, surgeons, nurses, pharmacists, case managers, social workers, and information technology experts gather and hold a sufficient number of meetings to make common, and shared decisions [10]. Instead of administering specific items such as in ERAS, the perioperative care in the PSH model adapts to the local hospital environment [20]. An individual PSH could have many elements of ERAS, but it focuses on the coordination of all hospital departments [20]. Some hospitals adopted the Lean Six Sigma method to launch the PSH. Lean, which comes from automobile company 'Toyota', is a method to reduce waste through rigorous standardization processes [22]. Six Sigma is a management innovation strategy that aims to maximize customer satisfaction by eliminating defects and minimizing deviation from the goal. All perioperative care pathways in the PSH model are aimed at achieving the best medical outcome with fewer deviations through Six Sigma and reducing waste by LEAN standardization [10].

Extensive education for patients and medical staff, goal-directed individual recovery plans, and standardized care are incorporated in every phase of the PSH protocol. All anesthesiologists and PSH perioperativists should share their plans for their patients. The acute pain team should check the pain levels of the patients on a daily basis and the PSH call system should be established for the continuity of care. For a smooth transition from hospital to home or other facility, a nurse navigator and a case manager work closely with the PSH team [10].

\section{Preoperative phase}

Once the decision for surgery has been taken, the patient is referred to the preoperative anesthetic clinic. Qualified, mid-level nurse practitioners work together with attending anesthesiologists with extensive knowledge and experience in perioperative care. In cases of patients with more complicated and complex medical conditions, more time is required to assess and optimize their status before surgery; this might necessitate comprehensive consultations involving other departments [11].

The preanesthetic clinic has established some evidence-based, and standardized protocols to identify certain preoperative patient conditions and appropriate management strategies, known to be safe and effective [23]. When compared to traditional preoperative investigations by surgeons, selective ordering by experienced anesthesiologists can significantly reduce the number of preoperative tests performed by between $29 \%$ and $55 \%[9,24]$.
This translates to a $40-59 \%$ relative reduction in total medical costs $[9,24]$.

In addition, a specific, early patient optimization strategy improves surgical outcomes [25]. Early engagement includes education for non-smoking and the use of beta-blockers, statins, or anticoagulation therapy, and the use of erythropoietin or iron, as necessary [11]. Patient education, engagement, and empowerment are very important in the pre-anesthetic period. Detailed information about perioperative flows around the surgery is given to the patients. This information contains the explanations for early postoperative feeding, early ambulation, pain control, and respiratory physiotherapy [8]. This can be accomplished in many ways such as personal counseling, pamphlets, and multimedia information. Reducing anxiety and educating patients about postoperative challenges are crucial for reducing the incidence of postoperative complications $[8,24]$.

Postoperative pain is also an important issue to manage. Patients are educated and empowered about their right to have effective analgesia. Achievement of preventive analgesia and avoidance of postoperative chronic pain development are also emphasized. This individual analgesic plan is developed preoperatively and is also directly communicated to the intraoperative anesthesiologist and postoperative pain service team [11]. If some interventions are needed, the pre-anesthetic clinic obtains prior patient consent; this lessens the stress for both the patient and the health care provider by obviating the need of making this choice on the day of the surgery itself. In addition, the pre-anesthetic clinic checks the written consent for the surgery and helps the surgeon complete the necessary preparations before surgery [11].

\section{Intraoperative phase}

To minimize case delay or cancellations, the PSH model tries not only to optimize the medical aspects of patient management but also to establish a strong consensus in the PSH team about the criteria for operating in an elective case [11]. The entire anesthesia team can deliver optimal, enhanced, and individualized anesthetic service to the patients through a fully integrated electronic medical record system [11].

Compared with other perioperative care models, the PSH model does not clarify the intraoperative anesthetic standards or decide which anesthetic techniques or medications are used. The PSH model respects and relies on the discretion of the anesthetic and pain service teams, but each center usually runs extensive education systems to enhance the knowledge of anesthetic updates and procedural advances. Some hospitals are operating a real-time electronic dashboard to allow all members of the anesthesiology team to know what the current protocol is and to benchmark their care [11]. Many intraoperative strat- 
egies, like ERAS, are available to lower the risk of postoperative complications and to enhance patient outcome. These include goal-directed fluid management [17], preference for regional anesthesia in complicated cases [8], and prophylaxis for postoperative nausea and vomiting $[8,18]$.

\section{Postoperative phase}

Surgical mortality rates vary widely across hospitals, varying between $3.5 \%$ and $6.9 \%$ [5]. Among the many possible causes for this, postoperative complications are known to have a greater impact on postoperative mortality and recovery than coexisting diseases or adverse events during surgery [26]. As one approach to reduce surgical mortality, health care providers focus on ways to reduce postoperative complications. However, even though there have been remarkable improvements in surgical technique and perioperative care, complication rates remain high (25-40\%) in the traditional perioperative care setting [5].

While there is no significant difference in the incidence of postoperative complications between hospitals, the mortality rates of patients with severe complications is 2-3-fold higher in hospitals with high mortality rates compared to those with low mortality $[5,27,28]$. The hospitals with high mortality rates have a much higher 'failure to rescue' than those with low mortality rates $(16.7 \%$ vs $6.8 \%)$, rather than a larger number of postoperative complications [27]. The main causes of this 'failure to rescue' are largely categorized into timely detection and appropriate treatment [29]. Most significant postoperative complications usually have some period of physiological deterioration in the ward [29]. Failure to timely detect and/or appropriately manage these physiologic deteriorations is a major factor in the 'failure to rescue' problem, including the postoperative setting. Therefore, although efforts should be made to reduce postoperative complications, there should also be a focus on timely detection of and appropriate management of these serious complications, once they occur [5].

The role of an anesthesiologist as a perioperativist is literally that of a surgical home doctor who manages the whole surgical pathway from preoperative preparation to postoperative recovery in the ward and up to one month post-discharge. In particular, the role in postoperative recovery management is expected to be close to the specialized surgical hospitalist attempted in United States (US) hospitals [30]. Because an anesthesiologist-perioperativist is sensitive to the patient's physiological declines and proficient in resuscitation, the PSH team's response to postoperative complications is expected to be very rapid and effective. This may improve outcomes by reducing the 'failure to rescue' rate and lowering the patient's surgical morbidity and mortality.

A number of traditional interventions, such as a routine use of nasogastric tube, preoperative bowel preparation in colorectal surgery, and fasting until bowel movements are restored, are still widely performed in Korea [31], even though they have been shown to be outdated, not evidence-based, and even harmful to the patient $[31,32]$. To overcome these outdated traditions, numerous interventions, with proven efficacy, have been developed. Among them, the leading postoperative care plan worldwide is the ERAS protocols. In one study, ERAS protocols shortened hospital stay by approximately 2.3 days and reduced complications by about $40 \%$ [33]. This resulted in a significant reduction in medical costs [33]. In the 'pay for value' system, which is the current trend, a perioperative model that reduces cost and increases quality must be of interest to physicians, patients, and hospital administrators. Several US hospitals that introduced PSH for the first time applied some of the ERAS protocols to their new models $[11,18]$. With a proven superiority in patient outcome and reduction of total medical costs through the ERAS protocol, the addition of continuity of care with the PSH model could upgrade the postoperative care model and make it more efficient. Researchers have shown interest in this unique idea of postoperative patient management by an anesthesiologist as a perioperativist.

Post-discharge care is continued to 30 days after discharge in the PSH model. Smooth transition from hospitalization to post-discharge continues to be a major factor in providing patients with confidence and security, and reducing emergency room visits and re-admission after discharge [11]. Both the PSH and ERAS protocols have common goals of increasing perioperative care quality and reducing medical costs, but there are differences in how they are implemented [19]. The ERAS protocol focuses on applying as many as 20 important items that have been proven and standardized by evidence before and after surgery $[8,33]$. However, there is still the possibility that effective cooperation and continuity of care between departments may not be achieved with the ERAS protocol alone. The PSH model has a larger conceptual framework over a longer period of time, and the appropriate application of specific perioperative care items is performed, depending on the unique circumstances of each institution [10,19]. The ERAS protocol is similar in most institutions, but PSH varies significantly across institutions [19].

\section{Perspective of the Surgeon}

PSH is a new model to increase the competence of care throughout whole operation, not to replace the surgeon's role in postoperative management [11]. From the standpoint of the surgeon, the PSH model has some creative value. In the relationship between the anesthesiologist and the patient, the patient is provided with a lot of information about the anesthesia before and after the operation and is positively influenced by the rela- 
tionship. The relationship between the surgeon and the anesthesiologist is also more cooperative and synergistic.

Although the demand for advanced care is increasing due to increased life expectancy and accompanying chronic diseases, the number of well-trained surgeons has not sufficiently increased to meet these needs. In recent years, special programs have been introduced in Korea to legally guarantee the working hours of interns and trainees in accordance with labor laws. Each hospital is struggling to find a way to compensate for a sudden drop in workforce, especially the night-shift workforce in the wards. In the surgical field especially, given the reality that a sufficient number of qualified surgeons are not available to match the operative load, postoperative care in the ward has become increasingly neglected. If the perioperative care team is expanded with an anesthesiologist who can communicate well with surgeons and knows the patient's perioperative condition, a more advanced surgical patient care can be achieved in the future.

\section{Perspective of Anesthesiologists}

To date, the full scope of perioperative care is not covered by any single departmental training. Surgeons are often not aware of the perioperative medical preparation and management of various problems associated with anesthesia. In addition, medical physicians are familiar with neither the anesthetic or surgical circumstances nor with acute pain management.

Anesthesiologists undergo special and extensive training to acquire intuitive insights and exceptional procedural skills ranging from preoperative preparation, intraoperative anesthetic management, and postoperative pain management to intensive care. They can manage the patients across the entire range of age and various comorbidities. Therefore, expansion into perioperative care by an anesthesiologist will be an effective way to provide high-quality health care and be very encouraging for the expansion of the specialty [11]. However, for this purpose, core knowledge and extended training for patient recovery in the ward, which is relatively lacking in the current anesthesiology training, should be supplemented considerably in the future [11]. In addition, the anesthesiologists should consider this flow not as an abandonment of traditional surgical anesthesia but as an expansion into a new area in a larger framework of anesthesiology, which includes subspecialties like critical care medicine and pain medicine [34]. The intraoperative anesthetic role will continue to be unique and intact [35]. This extended subspecialty may also be a new, attractive subject for medical students [11].

\section{Perspectives of Hospital Administrators}

In Korea, the need for a medical and surgical ward doctor (e.g., hospitalist) to compensate for the reduction in the working hours of trainees is being discussed. No single specialty can provide the full scope of perioperative primary care. Until now, co-management by medical doctors of perioperative care for complex surgical patients is common, especially for orthopedic, cardiothoracic, and neurosurgical patients. Most of this care has been provided by internal medicine trainees or fellows, and this lack of medical personnel is expected to be overcome by new physicians such as hospitalists. These hospitalist schemes have been already implemented in US. Several prospective studies of co-management by internal medicine hospitalists have shown very divergent results [36]. Further development and expansion of the role of internal medicine hospitalists in perioperative care has a major problem. Medical hospitalists often take care of patients with complex surgical histories mainly from an internal medicine point of view, without sufficient knowledge of and training on the various problems that arise after surgery and anesthesia [23].

Interestingly, there are some surgical hospitalist models in US and Canada. These models, composed of surgeons, were rated well in providing emergent surgical care, but showed weakness in resolving severe issues in patients with complex surgical histories [30].

As mentioned earlier, the new health care paradigm is changing to 'pay for value' $[15,37]$. To survive in this new medical setting, team-based effective care is needed rather than performance by individual physicians [38]. In 1998, the Institute of medicine National Roundtable on Health Care Quality concluded that health care quality problems stem from the trio of 'overuse, underuse, and misuse' of resources-a trio that remains paramount even today [39]. Evidence shows that patient-centered care not only improves clinical outcomes, quality of life, and patient satisfaction [40] but is also associated with a decrease in inappropriate health care utilization. The changes of payment paradigm such as value-based purchasing of health care [41,42], 'pay for performance' $[43,44]$, or bundled payments [45] are all powerful motivators to improve the quality of surgical care-particularly in the perioperative setting.

Patient-centered integrated care like PSH reduces the overuse, underuse, and misuse and improves outcome, resulting in excellent value at lower costs leading to a good business model [46] in perioperative medicine [47].

The PSH is a newly developed perioperative care system under the supervision of an anesthesiologist, which provides consistent, coordinated, and integrated care throughout the entire period, from the preoperative to the post-discharge phases. By providing evidence-based and standardized perioperative strategies to improve outcome for each hospital situation, patients can receive better medical care at a lower cost. This new model is expected to be a breakthrough in Korea's medical reality, which 
has many problems due to insufficient usage of surgical personnel. It will also be an attractive subspecialty for anesthesiologists.

\section{Acknowledgments}

The author would like to express deepest gratitude to Dr.
Rinehart in UC Irvine for his helpful advice.

\section{Funding Statement}

The present article was conducted by the research fund of Dankook University in 2015.

\section{References}

1. Berwick DM, Hackbarth AD. Eliminating waste in US health care. JAMA 2012; 307: 1513-6.

2. Grocott MP, Pearse RM. Perioperative medicine: the future of anaesthesia? Br J Anaesth 2012; 108: 723-6.

3. Kawashima Y, Takahashi S, Suzuki M, Morita K, Irita K, Iwao Y, et al. Anesthesia-related mortality and morbidity over a 5-year period in 2,363,038 patients in Japan. Acta Anaesthesiol Scand 2003; 47: 809-17.

4. Lagasse RS. Anesthesia safety: model or myth? A review of the published literature and analysis of current original data. Anesthesiology 2002; 97: 1609-17.

5. Ghaferi AA, Birkmeyer JD, Dimick JB. Variation in hospital mortality associated with inpatient surgery. N Engl J Med 2009; 361: $1368-75$.

6. Saunders DI, Murray D, Pichel AC, Varley S, Peden CJ. Variations in mortality after emergency laparotomy: the first report of the UK Emergency Laparotomy Network. Br J Anaesth 2012; 109: 368-75.

7. Moola S, Lockwood C. Effectiveness of strategies for the management and/or prevention of hypothermia within the adult perioperative environment. Int J Evid Based Healthc 2011; 9: 337-45.

8. Gustafsson UO, Scott MJ, Schwenk W, Demartines N, Roulin D, Francis N, et al. Guidelines for perioperative care in elective colonic surgery: Enhanced Recovery After Surgery $\left(\right.$ ERAS $^{\oplus}$ ) Society recommendations. Clin Nutr 2012; 31: 783-800.

9. Kehlet H, Wilmore DW. Multimodal strategies to improve surgical outcome. Am J Surg 2002; 183: 630-41.

10. Kain ZN, Vakharia S, Garson L, Engwall S, Schwarzkopf R, Gupta R, et al. The perioperative surgical home as a future perioperative practice model. Anesth Analg 2014; 118: 1126-30.

11. Vetter TR, Goeddel LA, Boudreaux AM, Hunt TR, Jones KA, Pittet JF. The perioperative surgical home: how can it make the case so everyone wins? BMC Anesthesiol 2013; 13: 6.

12. Pasternak LR. Preoperative testing: moving from individual testing to risk management. Anesth Analg 2009; 108: 393-4.

13. Fry DE, Pine M, Jones BL, Meimban RJ. The impact of ineffective and inefficient care on the excess costs of elective surgical procedures. J Am Coll Surg 2011; 212: 779-86.

14. McCulloch P, Nagendran M, Campbell WB, Price A, Jani A, Birkmeyer JD, et al. Strategies to reduce variation in the use of surgery. Lancet 2013; 382: 1130-9.

15. Miller HD. From volume to value: better ways to pay for health care. Health Aff (Millwood) 2009; 28: 1418-28.

16. White PF, Kehlet H, Neal JM, Schricker T, Carr DB, Carli F. The role of the anesthesiologist in fast-track surgery: from multimodal analgesia to perioperative medical care. Anesth Analg 2007; 104: 1380-96.

17. Cannesson M, Kain ZN. The role of perioperative goal-directed therapy in the era of enhanced recovery after surgery and perioperative surgical home. J Cardiothorac Vasc Anesth 2014; 28: 1633-4.

18. Garson L, Schwarzkopf R, Vakharia S, Alexander B, Stead S, Cannesson M, et al. Implementation of a total joint replacement-focused perioperative surgical home: a management case report. Anesth Analg 2014; 118: 1081-9.

19. Cannesson M, Kain Z. Enhanced recovery after surgery versus perioperative surgical home: is it all in the name? Anesth Analg 2014; 118: 901-2.

20. Chaurasia A, Garson L, Kain ZL, Schwarzkopf R. Outcomes of a joint replacement surgical home model clinical pathway. Biomed Res Int 2014; 2014: 296302.

21. Velanovich V, Rubinfeld I, Patton JH Jr, Ritz J, Jordan J, Dulchavsky S. Implementation of the national surgical quality improvement program: critical steps to success for surgeons and hospitals. Am J Med Qual 2009; 24: 474-9.

22. Teich ST, Faddoul FF. Lean management-the journey from toyota to healthcare. Rambam Maimonides Med J $2013 ; 4$ : e0007.

23. Berwick DM. What 'patient-centered' should mean: confessions of an extremist. Health Aff (Millwood) 2009; 28: w555-65.

24. Nygren J, Thacker J, Carli F, Fearon KC, Norderval S, Lobo DN. Guidelines for perioperative care in elective rectal/pelvic surgery: Enhanced Recovery After Surgery (ERAS ${ }^{\circledR}$ ) Society recommendations. Clin Nutr 2012; 31: 801-16.

25. Mayo NE, Feldman L, Scott S, Zavorsky G, Kim DJ, Charlebois P, et al. Impact of preoperative change in physical function on postoperative recovery: argument supporting prehabilitation for colorectal surgery. Surgery 2011; 150: 505-14.

26. Khuri SF, Henderson WG, DePalma RG, Mosca C, Healey NA, Kumbhani DJ. Determinants of long-term survival after major surgery and the adverse effect of postoperative complications. Ann Surg 2005; 242: 326-41.

27. Ghaferi AA, Birkmeyer JD, Dimick JB. Complications, failure to rescue, and mortality with major inpatient surgery in medicare patients. 
Ann Surg 2009; 250: 1029-34.

28. Ghaferi AA, Birkmeyer JD, Dimick JB. Hospital volume and failure to rescue with high-risk surgery. Med Care 2011; 49: $1076-81$.

29. Taenzer AH, Pyke JB, McGrath SP. A review of current and emerging approaches to address failure-to-rescue. Anesthesiology 2011; 115: 421-31.

30. Maa J, Carter JT, Gosnell JE, Wachter R, Harris HW. The surgical hospitalist: a new model for emergency surgical care. J Am Coll Surg 2007; 205: 704-11.

31. Joh YG, Lee JE, Yoo SH, Kim SH, Jeong GY, Chung CS, et al. The effects of a standardized postoperative enhanced recovery program after a laparoscopic colorectal resection in regard to patients' recovery and clinical outcomes. J Korean Soc Coloproctol 2010; 26: 225-32.

32. Miller TE, Thacker JK, White WD, Mantyh C, Migaly J, Jin J, et al. Reduced length of hospital stay in colorectal surgery after implementation of an enhanced recovery protocol. Anesth Analg 2014; 118: 1052-61.

33. Greco M, Capretti G, Beretta L, Gemma M, Pecorelli N, Braga M. Enhanced recovery program in colorectal surgery: a meta-analysis of randomized controlled trials. World J Surg 2014; 38: 1531-41.

34. Rock P. The future of anesthesiology is perioperative medicine. Anesthesiol Clin North America 2000; 18: 495-513.

35. Schubert A, Eckhout GV, Ngo AL, Tremper KK, Peterson MD. Status of the anesthesia workforce in 2011: evolution during the last decade and future outlook. Anesth Analg 2012; 115: 407-27.

36. Auerbach AD, Wachter RM, Cheng HQ, Maselli J, McDermott M, Vittinghoff E, et al. Comanagement of surgical patients between neurosurgeons and hospitalists. Arch Intern Med 2010; 170: 2004-10.

37. Kaplan RS, Porter ME. How to solve the cost crisis in health care. Harv Bus Rev 2011; 89: 46-52.

38. Lee TH. Turning doctors into leaders. Harv Bus Rev 2010; 88: 50-8.

39. Chassin MR, Galvin RW. The urgent need to improve health care quality. Institute of Medicine National Roundtable on Health Care Quality. JAMA 1998; 280: 1000-5.

40. Epstein RM, Fiscella K, Lesser CS, Stange KC. Why the nation needs a policy push on patient-centered health care. Health Aff (Millwood) 2010; 29: 1489-95.

41. Porter ME. What is value in health care? N Engl J Med 2010; 363: 2477-81.

42. Chee TT, Ryan AM, Wasfy JH, Borden WB. Current state of value-based purchasing programs. Circulation 2016; 133: $2197-205$.

43. Rosenthal MB, Dudley RA. Pay-for-performance: will the latest payment trend improve care? JAMA 2007; 297: 740-4.

44. Epstein AM. Pay for performance at the tipping point. N Engl J Med 2007; 356: 515-7.

45. Mayes R. Moving (realistically) from volume-based to value-based health care payment in the USA: starting with medicare payment policy. J Health Serv Res Policy 2011; 16: 249-51.

46. Lee A, Kerridge RK, Chui PT, Chiu CH, Gin T. Perioperative systems as a quality model of perioperative medicine and surgical care. Health Policy 2011; 102: 214-22.

47. Bohmer RM. Fixing health care on the front lines. Harv Bus Rev 2010; 88: 62-9. 